\title{
SYMMETRIC UNITS IN MODULAR GROUP ALGEBRAS
}

\author{
VICTOR BOVDI \\ Bessenyei Teachers College, 4401 Nyíregyháza, Hungary \\ L. G. KovÁcS \\ Australian National University, Canberra, ACT 0200, Australia \\ AND \\ S. K. SEHGAL \\ University of Alberta, Edmonton, Alberta T6G 2H1, Canada
}

\begin{abstract}
Let $p$ be a prime, $G$ a locally finite $p$-group, $K$ a commutative ring of characteristic $p$. The anti-automorphism $g \mapsto g^{-1}$ of $G$ extends linearly to an anti-automorphism $a \mapsto a^{*}$ of $K G$. An element $a$ of $K G$ is called symmetric if $a^{*}=a$. In this paper we answer the question: for which $G$ and $K$ do the symmetric units of $K G$ form a multiplicative group.
\end{abstract}

Let $G$ be a group, $K$ a commutative ring (with 1 ), and $U(K G)$ the group of units in the group algebra $K G$. The anti-automorphism $g \mapsto g^{-1}$ of $G$ extends linearly to an anti-automorphism $a \mapsto a^{*}$ of $K G$; this extension leaves $U(K G)$ setwise invariant. An element $a$ of $K G$ is called symmetric if $a^{*}=a$.

1991 Mathematics Subject Classification. Primary 16S34.

V. Bovdi is indebted to the University of Alberta for warm hospitality and generous support during a period when part of this work was done. His research was also supported by the Hungarian National Foundation for Scientific Research grant No. T 014279.

Typeset by $\mathcal{A M S}_{\mathcal{M}}-\mathrm{T}_{\mathrm{E}} \mathrm{X}$ 
It is an open problem to find the noncommutative $K G$ in which the symmetric units form a multiplicative group. Here we solve this under the assumption that $K$ has prime characteristic, $p$ say, and $G$ is a locally finite $p$-group. Our result is the following.

Theorem. Let $p$ be a prime, $K$ a commutative ring of characteristic $p$, and $G$ a nonabelian locally finite $p$-group. The symmetric units of $K G$ form a multiplicative group if and only if $p=2$ and $G$ is the direct product of an elementary abelian group and a group $H$ for which one of the following holds:

(i) $H$ has an abelian subgroup $A$ of index 2 and an element $b$ of order 4 such that conjugation by $b$ inverts each element of $A$;

(ii) $H$ is the direct product of a quaternion group of order 8 and a cyclic group of order 4 , or the direct product of two quaternion groups of order 8 ;

(iii) $H$ is the central product of the group $\left\langle x, y \mid x^{4}=y^{4}=1, x^{2}=[y, x]\right\rangle$ with a quaternion group of order 8 , the nontrivial element common to the two central factors being $x^{2} y^{2}$;

(iv) $H$ is isomorphic to one of the groups $H_{32}$ and $H_{245}$ defined below.

The relevant definitions are:

$$
\begin{gathered}
H_{32}=\langle x, y, u| x^{4}=y^{4}=1, \\
x^{2}=[y, x], \\
y^{2}=u^{2}=[u, x], \\
\left.x^{2} y^{2}=[u, y]\right\rangle, \\
H_{245}=\langle x, y, u, v| x^{4}=y^{4}=[v, u]=1, \\
x^{2}=v^{2}=[y, x]=[v, y], \\
y^{2}=u^{2}=[u, x], \\
\left.x^{2} y^{2}=[u, y]=[v, x]\right\rangle .
\end{gathered}
$$

Note that in case (i) all elements of $H$ outside $A$ have order 4 and so any one of them can serve as $b$. The list of groups in this theorem is part of the 
list in Theorem 1.2 of Bovdi and Kovács [1], and the proof relies heavily on Lemma 1.4 of that paper.

The proof of the Theorem will occupy the rest of this note.

Set $S=\left\{t \mid t \in G, t^{2}=1\right\} \cup\left\{g+g^{-1} \mid g \in G, g^{2} \neq 1\right\}$, and note that the symmetric elements of $K G$ are precisely the $K$-linear combinations of the elements of $S$. Like the fixed points of any anti-automorphism of any group, the symmetric units form a subgroup in $U(K G)$ if and only if they commute with each other. It is well known that once $K$ is of characteristic $p$ and $G$ is a locally finite $p$-group, the augmentation ideal of $K G$ is locally nilpotent, and so every element congruent to 1 modulo this ideal is a unit. In particular, $-1+g+g^{-1}$ is a symmetric unit in $K G$ whenever $g \in G$. Of course, $t$ is a symmetric unit whenever $t \in G$ and $t^{2}=1$. This proves that the symmetric units form a multiplicative group if and only if every pair of elements of $S$ commutes.

In particular, for a given $p$ this issue is independent of the choice of $K$. It will be convenient to call a locally finite $p$-group good if every pair of elements in $S$ commutes (say, in $K G$ with $K=\mathbb{Z} / p \mathbb{Z}$ ). Note that all abelian groups are good, all subgroups of good groups are good, and that a locally finite $p$-group is good if all its 2 -generator subgroups are good.

In a good group, any two involutions (that is, elements of order 2 ) commute. If $g$ and $t$ are as in the definition of $S$, then the only way $t$ and $g+g^{-1}$ can commute is if $g^{t}\left(=t^{-1} g t\right)$ is either $g$ or $g^{-1}$. In the second case the subgroup $\langle g, h\rangle$ generated by $g$ and $t$ is a nonabelian dihedral group and therefore contains noncommuting involutions. This proves that in a good group every involution is central.

Next we prove that if $g, h$ are noncommuting elements in a good group $G$, then there exist $x, y$ in $G$ such that $\langle g, h\rangle=\langle x, y\rangle$ and $x^{y}=x^{-1}$. To this end, note that any two of $g, h, g h$ generate the nonabelian group $\langle g, h\rangle$, so by the previous paragraph none of them can have square 1 . On the other 
hand, $g+g^{-1}$ and $h+h^{-1}$ commute: thus

$$
g h+g h^{-1}+g^{-1} h+g^{-1} h^{-1}=h g+h g^{-1}+h^{-1} g+h^{-1} g^{-1} .
$$

If $g h$ occurs more than once on the left hand side, we must have $g h=g^{-1} h^{-1}$, so $x=g h, y=h$ will do. Otherwise $g h$ must equal one of the summands on the right hand side. That summand cannot be $h g$, for $g$ and $h$ do not commute; nor can it be $h^{-1} g^{-1}$, for $(g h)^{2} \neq 1$. Thus either $g h=h g^{-1}$, in which case $x=g, y=h$ will work, or $g h=h^{-1} g$, and then we can take $x=h, y=g$.

This already shows that the prime $p$ involved in a nonabelian good group can only be 2 .

The point established in the second last paragraph can be taken further: in those circumstances, $x$ and $y$ can be chosen so that the order of $y$ is 4 . To see this, note first that $(x y)^{-1}=x y^{-1} \neq x y$, hence $x y+x y^{-1}$ and $y+y^{-1}$ must commute. Given that the characteristic is 2 , this leads to $x\left(y^{2}+y^{-2}\right)=x^{-1}\left(y^{2}+y^{-2}\right)$. If the cosets $x\langle y\rangle$ and $x^{-1}\langle y\rangle$ are different, this forces $y^{2}+y^{-2}=0$, that is, $y^{4}=1$. If $x\langle y\rangle=x^{-1}\langle y\rangle$, it is easy to deduce that this is the only nontrivial coset of $\langle y\rangle$ in $\langle x, y\rangle$. Groups of 2-power order with a cyclic subgroup of index 2 are well known (see for example Section 109 in Burnside's book [2]); the nonabelian groups of this kind with all involutions central are precisely the generalized quaternion groups. (We count the quaternion group of order 8 among the generalized quaternion groups.) Of course, each generalized quaternion group can be generated by a pair of elements $x, y$ such that $x^{y}=x^{-1}$ and $y^{4}=1$.

We sum up these conclusions in the following.

Lemma 1. If $G$ is a nonabelian good group, then $p=2$ and each nonabelian 2-generator subgroup of $G$ is either a generalized quaternion group or a semidirect product

$$
C_{2^{m}} \rtimes C_{4}=\left\langle x, y \mid x^{2^{m}}=y^{4}=1, x^{y}=x^{-1}\right\rangle
$$


with $m \geq 2$.

If $m>2$ then there are other semidirect products that might be called $C_{2^{m}} \rtimes C_{4}$, but we shall always mean this one.

Lemma 2. If $G$ is a nonabelian good group and the exponent of $G$ is not 4 , then $G$ has an abelian subgroup $A$ of index 2 and an element $b$ of order 4 such that conjugation by $b$ inverts each element of $A$.

Proof. Set $A=\left\langle a \in G \mid a^{4} \neq 1\right\rangle$. Suppose first that $A$ is nonabelian. Then there are noncommuting elements $g, h$ in $A$ whose orders are greater than 4 . In a generalized quaternion group, all elements of order greater than 4 lie in one cyclic subgroup, so $\langle g, h\rangle$ cannot be a generalized quaternion group. In a $C_{2^{m}} \rtimes C_{4}$, all elements outside $\left\langle x, y^{2}\right\rangle$ have order 4 , so $\langle g, h\rangle$ cannot be a $C_{2^{m}} \rtimes C_{4}$ either. This contradiction to Lemma 1 proves that $A$ must be abelian.

Let $b$ be any element of $G$ outside $A$ : by the definition of $A$, then $b^{4}=1$. If $a^{4} \neq 1$, then $a$ and $b$ cannot commute (else we would have $(a b)^{4} \neq 1$ and then $a, a b \in A, b \in A$ would follow, contrary to the choice of $b$ ). In a generalized quaternion group or in a $C_{2^{m}} \rtimes C_{4}$, an element of order greater than 4 can only be conjugate to itself or to its inverse, so Lemma 1 implies that $a^{b}=a^{-1}$. It follows that $b$ inverts every element of $A$. That includes $b^{2}$, so $b^{4}=1$, and as all involutions are central we cannot have $b^{2}=1$.

It follows from Lemma 1 that if $G$ is a nonabelian good group of exponent 4 and if $g, h \in G$, then $\left\langle g^{2}\right\rangle$ is central and $\langle g, h\rangle /\left\langle g^{2}\right\rangle$ is abelian or dihedral. Under somewhat weaker hypotheses, Lemma 1.4 of [1] asserts that $G$ is a direct product of an elementary abelian group and a group $H$ such that either $H$ satisfies one of the conditions (i)-(iv) of our Theorem, or $H$ is an extraspecial 2 -group, or $H$ is the central product of an extraspecial 2 -group with a cyclic group of order 4 . All central products of this kind and all extraspecial 2-groups except the quaternion group contain noncentral involutions, while the quaternion group satisfies condition (i). This completes the proof of the 'only if' part of our Theorem. 
The proof of the 'if' part is much easier. The definition of good group directly yields that the direct product of an elementary abelian 2-group and a good 2-group is always good: thus it suffices to check that if $p=2$ and one of the conditions (i)-(iv) holds then $H$ is good.

Consider case (i) first. We have already remarked that in this case all involutions of $H$ lie in $A$, so they are all central. If $g \in A$, then $g+g^{-1}$ commutes with every element of $H$ and is therefore central in $K H$. If both $g$ and $h$ are elements of $H$ outside $A$, one can play the role of $b$ and the other can be written as $a b$ with $a \in A$, and $\left(b+b^{-1}\right)^{2}=0$ implies that $\left(b+b^{-1}\right)\left((a b)+(a b)^{-1}\right)=0=\left((a b)+(a b)^{-1}\right)\left(b+b^{-1}\right)$. Thus in this case $H$ is good.

In the other three cases $H$ has exponent 4 and we know (from O'Brien's Lemma 4.1 in [1], or by direct calculation) that all involutions in $H$ are central. We can also see that the Frattini subgroup $\left\langle h^{2} \mid h \in H\right\rangle$ of $H$ has order 4. Thus if $g, h \in H$, then $\langle g, h\rangle$ has order at most 16. The groups of order dividing 16 are well known (see for example Section 118 in [2]); there are only two 2 -generator nonabelian groups of exponent 4 among them in which all involutions are central, and both of those satisfy condition (i). Thus by the previous paragraph $\langle g, h\rangle$ is good, and so $g+g^{-1}$ commutes with $h+h^{-1}$. We conclude that $H$ is good in each of these cases.

This completes the proof of the Theorem.

\section{REFERENCES}

1. Victor Bovdi and L. G. Kovács, Unitary units in modular group algebras, Manuscripta Math. 84 (1994), 57-72.

2. W. Burnside, Theory of groups of finite order, second edition, Cambridge University Press, 1911, reprinted by Dover, 1955. 